\title{
Zusammenspiel zwischen Diskurs und Syntax im Erwerb des Deutschen als Zweitsprache in der Kindheit
}

\section{Interplay of discourse and syntax in the acquisition of German as a second language in childhood}

\begin{abstract}
AвSTRACT. The goal of this study is to investigate the interplay of discourse and syntax, as it can be observed in the use of null objects, in the language development of a child who starts to acquire German at the age of nine years in a naturalistic setting. The study shows that children at such an age can profit from the knowledge already acquired in their first language. After nine months of exposure, the child under study uses null objects similarly to monolingual children. The acquisition of null objects in a second language can be impeded at an early stage, however, due to the different syntactic restraints for the use of null objects in the first and second languages.
\end{abstract}

KEYwords: German as a second language, null objects, discourse, syntax.

SCHLÜsSELWÖRTER: Deutsch als Zweitsprache, Nullobjekte, Diskurs, Syntax.

\section{EINFÜHRUNG}

Der Gebrauch jeder menschlichen Sprache verlangt vom Sprecher, die syntaktischen Informationen in einen adäquaten Diskursrahmen zu integrieren. Mit dem Begriff „Diskurs“ wird hier eine Einheit verstanden, die größer als ein Satz ist, und die die Kriterien der Kohäsion sowie der Kohärenz erfüllt ${ }^{1}$.

Die Untersuchungen von Diskurs werden vor allem als Teilbereich der Pragmatik verstanden, weil ihr Gegenstand der Gebrauch der Äußerungen während der Kommunikation ist. Wenn jedoch die formale Abhängigkeitsstruktur untersucht wird, die die Satzgrenzen einerseits überschreitet und die andererseits den Satzaufbau und die Wahl der sprachlichen Formen beeinflusst, dann müssen zur Erklärung solcher Phänomene sowohl die Pragmatik als auch die Syntax herangezogen werden.

${ }^{1}$ Im Rahmen des Artikels kann nicht auf die vielen Bedeutungen des Begriffs „Diskurs“ eingegangen werden. 
Eines der Phänomene, bei denen man das Zusammenspiel von syntaktischen und pragmatischen Regeln sehr gut sehen kann, ist der Gebrauch der Nullargumente. Subjekte und Objekte können in mehreren Sprachen phonetisch nicht realisiert werden. Die Möglichkeit unterliegt aber sowohl pragmatischen als auch syntaktischen Einschränkungen. Die Bedingungen, unter denen sie zulässig sind, können von Sprache zu Sprache unterschiedlich sein.

\section{NULLOBJEKTE IM DEUTSCHEN UND POLNISCHEN}

Referentielle Argumente können in jeder Sprache unterschiedlich realisiert werden. Die Formen der referentiellen Ausdrücke mögen von indefiniten über definite Nominalphrasen, starke und schwache Pronomen bis hin zu Nullargumenten reichen.

Gundel, Hedberg und Zacharski (1993) schlagen eine Hierarchie der Möglichkeiten des kognitiven Status eines referentiellen Ausdrucks vor (siehe Tabelle 1). Der kognitive Status spiegelt die Zugänglichkeit des Referenten im Redeuniversum wider und beeinflusst in hohem Grade die Wahl der entsprechenden Form des referentiellen Ausdrucks (vgl. Fukumura \& van Gompel 2011; siehe auch Sopata 2017 für die Zusammenfassung der Diskussion).

Tabelle 1. Givenness-Hierarchie

in focus $>$ activated $>$ familiar $>$ uniquely identifiable $>$ referential $>$ type identifiable

In Fokus > Aktiviert $>$ Vertraut $>$ Identifizierbar $>$ Referentiell $>$ Typ-Identifizierbar

Quelle: Gundel et al. (1993).

Nach Gundel et al. (1993) determiniert der kognitive Status des Referenten den Gebrauch einer gegebenen Form des referentiellen Ausdrucks. Die Stelle des Arguments in der Gegebenheitshierarchie, die visuelle Präsenz des Diskursreferenten und seine Salienz im Diskurs beeinflussen den kognitiven Status des gegebenen Referenten. Wenn das Argument im Kopf des Sprechers und des Hörers aktiviert ist, dann kann es in einigen Sprachen als Nullargument realisiert werden.

Die Form der referentiellen Argumente wird jedoch auch durch ihre syntaktischen Eigenschaften determiniert. Nach Huang (1984), Holmberg (2005) und Sigurðsson (2011) kann man in der Hinsicht von drei Klassen von Nullargumenten reden (vgl. auch Sopata 2017):

- Nullargumente, die mit der Subjekt-Verb oder Objekt-Verb-Kongruenz verbunden sind, wie beispielsweise Nullsubjekte in romanischen Sprachen und im Polnischen, oder Nullobjekte in Pashto (siehe Beispiel 2), 
- Nullargumente, die mit der Topik-Position verbunden sind, d.h. solche, die nur am Satzanfang realisiert werden können, wo die in der Satzstruktur hervorgehobenen Elemente vorkommen, wie beispielsweise Nullsubjekte und Nullobjekte im Deutschen (siehe Beispiel 1),

- Nullargumente, die satzextern konditioniert werden, wie beispielsweise Nullsubjekte und Nullobjekte im Chinesischen sowie Nullobjekte im Polnischen (siehe Beispiel 2).

Im Deutschen können die Nullargumente nur in der Topik-Position auftreten. Auf die Frage eines Gesprächspartners im Beispiel 1 können mehrere Antworten in einem Gespräch gegeben werden (1a-1d). Man kann eine volle Nominalphrase oder ein Pronomen gebrauchen (siehe 1a). Das Objekt, das durch eine volle Nominalphrase oder ein Pronomen ausgedrückt wird, kann in die sog. Topik-Position, d.h. an den Satzanfang bewegt werden (siehe 1b). Wenn das Objekt sich am Satzanfang, in der sog. Topik-Position befindet, dann kann die Form des Nullobjekts benutzt werden (siehe 1c). Im Satzinneren kann das Objekt jedoch nicht als Null ausgedrückt werden, obwohl es im Kopf des Sprechers und des Hörers aktiviert ist (siehe 1d).

(1) Person 1: Wo steht dein Auto?

Person 2:

Ich habe das Auto/es verkauft.

Das Auto/Das hab ich verkauft.

_hab ich verkauft.

*Ich habe_verkauft.

Im Polnischen muss das Objekt im Kopf des Sprechers auch aktiviert werden, und den kognitiven Status des vertrauten Arguments haben, um als Null realisiert zu werden. Seine Aktivierung kann beispielsweise durch sein Auftreten in der voranstehenden Äußerung eines Gesprächspartners erfolgen (siehe Beispiel 2).

$\begin{array}{ll}\text { Person 1: } & \text { Gdzie stoi twój samochód? } \\ & \text { 'Wo steht dein Auto?' } \\ \text { Person 2: } & \quad \text { _Sprzedałam_. } \\ & \text { 'habe verkauft__ } \\ & \text { 'Ich habe das Auto verkauft.' }\end{array}$

Kein Nullobjekt kann gebraucht werden, wenn das Argument nicht aktiviert ist (siehe Beispiel 3): 


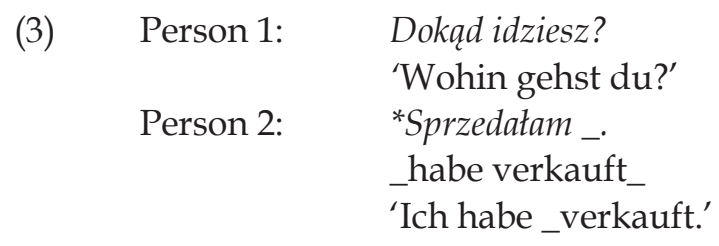

Die Aktivierung des Arguments kann auch durch den situativen Kontext erfolgen, ohne dass es im vorhergehenden Diskurs auftritt (siehe Beispiel 4):

$$
\begin{array}{ll}
\text { Person 1: } & \text { Co z tym? } \\
& \text { 'Was damit?' }
\end{array}
$$

(Auf dem Parkplatz deutet der Sprecher auf einen leeren Platz hin, an dem vorher das Auto des Gesprächspartners stand)

$$
\begin{array}{ll}
\text { Person 2: } & \text { Sprzedatam _. } \\
& \text { 'I } \text { habe verkauft__ habe das Auto verkauft.' }
\end{array}
$$

Wie das Beispiel 2 verdeutlicht, unterscheiden sich die syntaktischen Einschränkungen für den Nullobjektgebrauch im Polnischen (vgl. Mykhaylyk \& Sopata 2016; Sopata 2016) von denen im Deutschen. Der wichtigste Unterschied liegt darin, dass die Objekte im Polnischen auch im Satzinneren als Null realisiert werden können. Sie sind also nicht wie im Deutschen an die Topik-Position gebunden. Die Nullobjekte sind im Polnischen satzextern bedingt und werden durch den Diskurs interpretiert.

Anzumerken ist noch, dass von den Nullobjekten im Polnischen nur bei den Verben, die im perfektiven Aspekt verwendet werden, die Rede sein kann, weil nur sie obligatorisch mit einem Objekt auftauchen. Wenn die Verben im imperfektiven Aspekt gebraucht werden, dann können sie in der intransitiven Variante, also ohne direktes Objekt vorkommen. Sie drücken dann weder Handlungsabschluss noch Zustandswechsel aus (vgl. Kotin 2003: 199f.). Im Beispiel $5 a$ ist die Verwendung des Verbs im perfektiven und in $5 \mathrm{~b}$ im imperfektiven Aspekt zu sehen:

$$
\begin{aligned}
& \text { Person 1: } \quad \text { Co przeczytataś? } \\
& \text { 'Was hast du gelesen }{ }_{\text {perfektiv? }} \text { ' } \\
& \text { Person 2: } \quad \text { Przeczytatam artykut. } \\
& \text { 'Ich habe den Artikel gelesen } \text { perfektiv' } \text { ' }
\end{aligned}
$$


$(5 b)$

$\begin{array}{ll}\text { Person 1: } & \text { Co robiłaś? } \\ & \text { 'Was hast du gemacht } \text { imperfektiv }^{\text {? }} \\ \text { Person 2: } & \text { Czytatam. } \\ & \text { 'Ich habe gelesen } \\ & \end{array}$

\section{NULLOBJEKTE IM ERWERB DES DEUTSCHEN ALS ERSTSPRACHE}

Die Aufgabe, die syntaktischen und pragmatischen Aspekte der Sprache zu koordinieren, ist eine Herausforderung für Kinder, die ihre Erstsprache erwerben. Die obligatorischen morpho-syntaktischen Elemente werden manchmal durch die Kinder im Erstspracherwerb ausgelassen, was von der noch nicht perfekten Meisterung der Phänomene an der Schnittstelle Syntax-Diskurs zeugt. Die im Erstspracherwerb oft ausgelassenen Elemente sind Subjekte, Objekte und Artikelwörter.

Hamann (1996: 180) hat den Gebrauch der Nullobjekte im monolingualen Erwerb des Deutschen untersucht. Das erste von ihr untersuchte Kind wurde vom Alter 3;4 bis zum Alter 3;7 und das andere Kind vom Alter 3;1 bis zum Alter 3;4 aufgenommen. Haman (1996: 174) hat die Aufnahmen in vier Entwicklungsstufen zusammengefasst. Die Anzahl von Nullobjekten in den Daten von beiden Kindern - Christoph und Elisa - werden zu vier Zeitpunkten in den Tabellen 2 und 3 dargestellt. Die Bezeichnungen P1-P4 in den Tabellen beziehen sich auf die Phasen der Sprachentwicklung von Christoph und Elisa, die von Hamann identifiziert worden sind (vgl. auch Sopata 2017).

Tabelle 2. Nullobjekte in den Daten von Christoph

\begin{tabular}{|c|c|c|}
\hline Zeit & $\begin{array}{c}\text { Nullobjekte in der Topik-Position } \\
\text { [in \%] }\end{array}$ & $\begin{array}{c}\text { Nullobjekte in der Nicht-Topik-Position } \\
\text { [in \%] }\end{array}$ \\
\hline P1 & 28,5 & 2,7 \\
\hline P2 & 44,4 & 2,5 \\
\hline P3 & 31,8 & 1,3 \\
\hline P4 & 50,0 & 5,0 \\
\hline
\end{tabular}

Quelle: Hamann (1996).

Tabelle 3. Nullobjekte in den Daten von Elisa

\begin{tabular}{|c|c|c|}
\hline Zeit & $\begin{array}{c}\text { Nullobjekte in der Topik-Position } \\
\text { [in \%] }\end{array}$ & $\begin{array}{c}\text { Nullobjekte in der Nicht-Topik-Position } \\
\text { [in \%] }\end{array}$ \\
\hline P1 & 28,5 & 2,3 \\
\hline P2 & 13,0 & 0,5 \\
\hline P3 & 42,0 & 1,6 \\
\hline P4 & 20,0 & 1,5 \\
\hline
\end{tabular}

Quelle: Hamann (1996). 
Die Tabellen zeigen, dass monolinguale Kinder, die Deutsch erwerben, häufig von der Möglichkeit Gebrauch machen, Nullobjekte in der Topik-Position zu benutzen (vgl. auch Sopata 2017). Da solche Strukturen auch in der Erwachsenensprache vorkommen, gelten sie als normgerecht. Darüber hinaus lassen die Kinder aber auch Objekte in der Nicht-Topik-Position aus, in der die Erwachsenensprache keine Nullobjekte zulässt. Die Anzahl dieser Nullobjekte ist jedoch niedrig (vgl. auch Sopata 2017).

Mehrere empirische und theoretische Studien zur Wahl der Form der referentiellen Argumente im Erstspracherwerb sind in den letzten Jahren entstanden. Im Zentrum des Interesses standen meistens Auslassungen von klitischen Objekten (z.B. Clark 1985; Costa, Lobo \& Silva 2012; Jakubowicz, Müller, Kang, Riemer \& Rigaut 1996; Jakubowicz, Müller, Riemer \& Rigaut 1997; Müller \& Hulk 2001; Pérez-Leroux, Pirvulescu \& Roberge 2008; Wexler, Gavarro \& Torrens 2004).

In der Erstspracherwerbsforschung tauchen mehrere Hypothesen auf, die zum Ziel haben, die im Laufe des Erstspracherwerbs vorkommenden Nullobjekte zu erklären. Einige Ansätze verbinden das Auslassen der obligatorischen Objekte einerseits mit den Einschränkungen des kognitiven Systems der Kinder oder andererseits mit den Unterschieden zwischen den mentalen Grammatiken bei Kindern und Erwachsenen, die auf verschiedenen Ebenen auftauchen können. Ansätze, die nach einer syntaktischen Erklärung der Auslassungen suchen, verbinden sie oft mit der Komplexität der Verarbeitung der klitischen Elemente (siehe zum Beispiel Hamann, Rizzi \& Frauenfelder 1996; Gruter 2006; Gavarró, Torrens \& Wexler 2010). Die Ansätze, die nach einer pragmatischen Erklärung dieses Phänomens suchen, verbinden die Auslassungen mit verschiedenen Diskurseigenschaften (siehe zum Beispiel Matthews, Lieven, Theakston \& Tomasello 2006; Hughes \& Allen 2013). Ein Überblick über die unterschiedlichen Ansätze kann in Castilla \& Pérez-Leroux (2010) oder in Gavarró et al. (2010) gefunden werden.

\section{NULLOBJEKTE IM ERWERB DES DEUTSCHEN ALS ZWEITSPRACHE IN DER KINDHEIT}

In der vorliegenden Studie wird der Gebrauch der Nullobjekte bei einem Kind untersucht, das Deutsch als seine Zweitsprache unter natürlichen Bedingungen erwirbt. Die Untersuchung soll auf folgende Fragen Antwort geben:

1. Wie sieht die Sprachentwicklung im Bereich der Nullobjekte bei dem Kind aus, das den Erwerb der Zweitsprache mit 9 Jahren angefangen hat?

2. Kann das Kind daraus Nutzen ziehen, dass es das Zusammenspiel der syntaktischen und der pragmatischen Einschränkungen beim Gebrauch der Nullobjekte in ihrer Erstsprache schon erworben hat? 
3. Wird der Erwerb der syntaktischen Einschränkungen für den Gebrauch der Nullobjekte in der Zweitsprache dadurch erschwert, weil sie sich von denen in der Erstsprache unterscheiden?

Um auf die oben genannten Fragen eine Antwort zu finden, werden Daten von einem Kind - Ala - über einen Zeitraum von einem Jahr gesammelt. Ala ist in Polen geboren und im Alter von 9 Jahren nach Deutschland gekommen. Ihre Erstsprache sowie die ihrer Eltern ist Polnisch. Zum Zeitpunkt der Ankunft in Deutschland spricht Ala kein Deutsch. Seit dem Alter von 9;1 besucht das Mädchen eine Grundschule. In der Schule bleibt sie ca. 5 Stunden täglich. Ihre Erstsprache ist sehr gut entwickelt. Die Aufnahmen von Ala werden in monatlichem Abstand gemacht. Die erste Aufnahme wird zum Zeitpunkt eines abgeschlossenen Kontaktmonats (KM) zu Deutsch gemacht (zu weiteren Details siehe Sopata 2009).

Alas Gebrauch der Nullobjekte in der Nicht-Topik-Position kann der Abbildung 1 entnommen werden. In einigen Monaten ist der Anteil der Nullobjekte an allen Objekten in der Nicht-Topik-Position in Alas Daten ziemlich hoch. In KM5, KM6 und KM7 überschreitet er 20\%. In anderen Aufnahmen liegt der Wert um $10 \%$.

Ala

Nullobjekte in der Nicht-Topic-Position

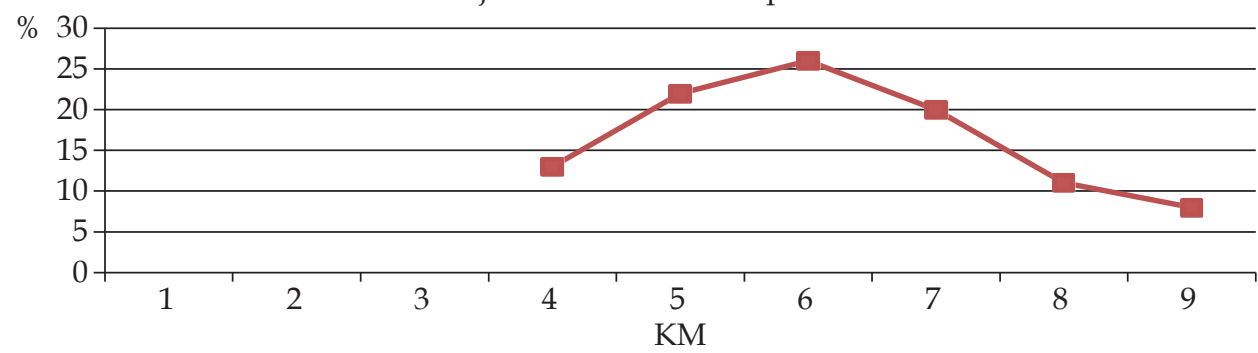

Abbildung 1. Gebrauch der Nullobjekte in der Nicht-Topik-Position in aufeinander folgenden Kontaktmonaten - Ala

Wenn wir Alas Daten mit denen der monolingualen Kinder vergleichen, die Deutsch als ihre Erstsprache erwerben, dann ist ein deutlicher Unterschied zwischen den Sprachprofilen zu sehen. Der Vergleich des Gebrauchs der Nullobjekte in der Nicht-Topik-Position von Ala einerseits und von den monolingualen Kindern aus Hamanns Studie andererseits wird in der Abbildung 2 dargestellt:

Der Abbildung kann entnommen werden, dass der Gebrauch der Nullobjekte in der Nicht-Topik-Position in Alas Daten im Allgemeinen viel höher als in Christophs und Elisas Daten liegt. Im monolingualen Erwerb des Deutschen 
liegt der Anteil der Nullobjekte an allen Objekten in der Nicht-Topik-Position im Allgemeinen bei $2 \%$ und im Erwerb des Deutschen als Zweitsprache in der Kindheit liegt der Wert bei $17 \%$.

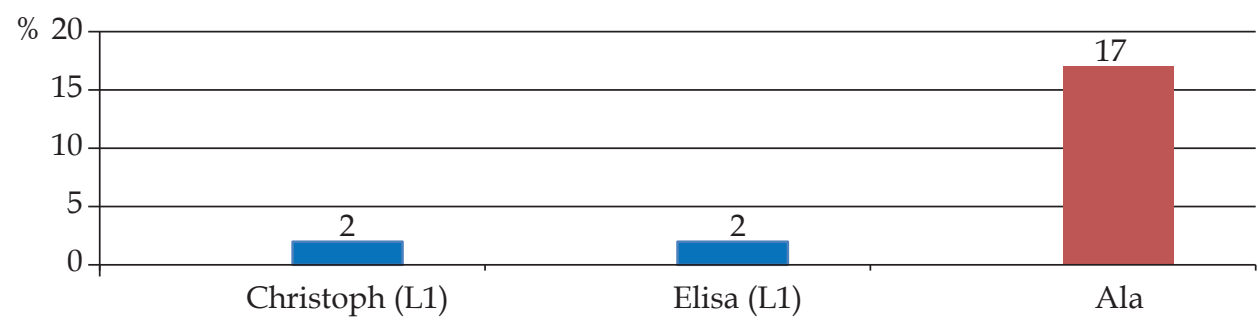

Abbildung 2. Gebrauch der Nullobjekte in der Nicht-Topik-Position - Christoph (L1), Elisa (L1) und Ala (L2)

Die Situation sieht etwas anders aus, wenn man die Ergebnisse des Gebrauchs der Nullobjekte in der Topik-Position ansieht. Die Abbildung 3 stellt den Gebrauch der Nullobjekte in der Topik-Position in Alas Daten vor.

Ala

Nullobjekte in der Topic-Position

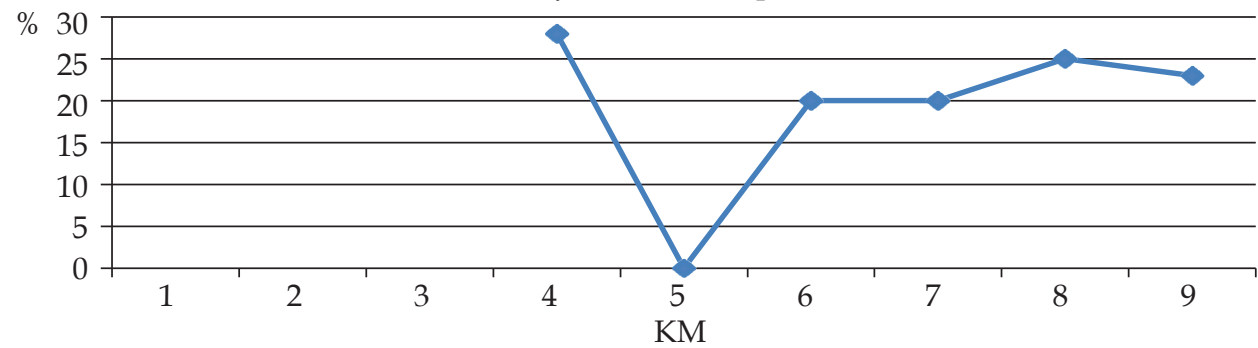

Abbildung 3. Gebrauch der Nullobjekte in der Topik-Position in aufeinander folgenden Kontaktmonaten - Ala

Der Abbildung 3 kann man entnehmen, dass Ala mehrere Nullobjekte in der Topik-Position in den meisten Aufnahmen benutzt. Der Anteil der Nullobjekte an allen Objekten in der Topik-Position beträgt zwischen 20\% und 30\%. Nur in einer Aufnahme liegt der Wert bei 0\%. Interessanterweise kommen die TopikStrukturen in KM1-KM3 bei Ala gar nicht vor, was auf die niedrigere Stufe der Sprachentwicklung zu dieser Zeit bei Ala zurückzuführen ist.

Alas Sprachentwicklung unterscheidet sich nicht gravierend von dem der monolingualen Kinder. Ein Vergleich von Alas, Christophs und Elisas Daten ist in der Abbildung 4 zu sehen. 


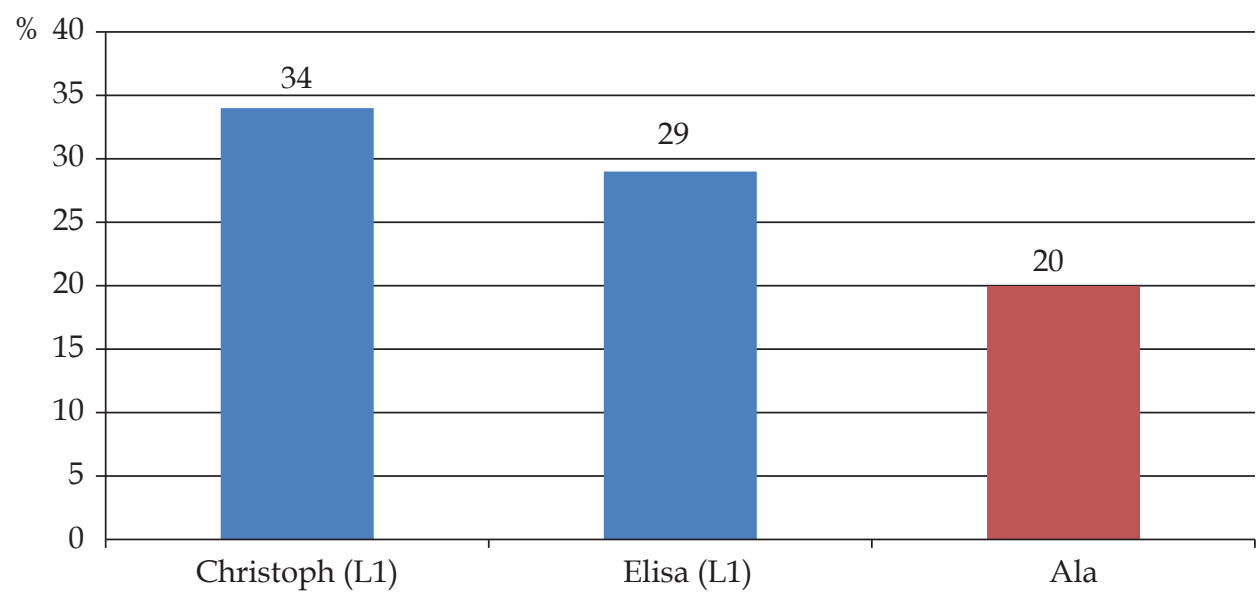

Abbildung 4. Gebrauch der Nullobjekte in der Topik-Position - Christoph (L1), Elisa (L1) und Ala (L2)

Die Abbildung 4 zeigt, dass der Anteil der Nullobjekte an allen Objekten in der Topik-Position im Allgemeinen bei einem monolingualen Kind 34\% bei dem anderen $29 \%$ beträgt. Der Wert liegt bei Ala, die Deutsch als ihre Zweitsprache erwirbt, bei $17 \%$. Es ist demnach ein klarer Unterschied. Wenn wir jedoch bedenken, dass es sich bei den Werten um ganz allgemeine Durchschnittswerte in allen Aufnahmen handelt, dann scheint der Unterschied nicht sehr groß zu sein.

Zusammenfassend kann man sagen, dass die Entwicklung des Deutschen bei dem Kind, das den Erwerb der Zweitsprache im Alter von 9 Jahren angefangen habt, sich von den Sprachprofilen der Kinder unterscheidet, die Deutsch als ihre Erstsprache erwerben. In der Hinsicht auf den Gebrauch der Nullobjekte in der Nicht-Topik-Position ist ein deutlicher Unterschied zwischen dem bilingualen und den monolingualen Kindern zu sehen. Wenn man die Ergebnisse des Gebrauchs der Nullobjekte in der Topik-Position genau betrachtet, dann sieht man auch einen Unterschied zwischen den Sprachprofilen der Kinder. Der Unterschied scheint jedoch in diesem Bereich etwas kleiner zu sein.

\section{SCHLUSSFOLGERUNGEN}

Auf Grund der im Rahmen der Studie durchgeführten Untersuchungen kann man folgende Antworten auf die oben gestellten Fragen geben. In Bezug auf die Frage Nr. 1 muss man feststellen, dass sich die Sprachentwicklung im Bereich der Nullobjekte von dem Kind, das den Erwerb der Zweitsprache mit 9 Jahren begonnen hat, von der der monolingualen Kinder gravierend unterscheidet. Ala 
benutzt einerseits Nullobjekte in der Nicht-Topik-Position viel häufiger als jene Kinder, die Deutsch als ihre Erstsprache erwerben. Andererseits gebraucht sie die Nullobjekte in der normgerechten Topik-Position etwas seltener als monolinguale Kinder.

Bei der Beantwortung der zweiten Forschungsfrage kann festgehalten werden, dass das Kind nach einiger Zeit daraus Nutzen ziehen kann, dass es die Koordination der syntaktischen und der pragmatischen Einschränkungen beim Gebrauch der Nullobjekte in ihrer Erstsprache schon erworben hat. Der Erwerb des Deutschen als Zweitsprache verläuft bei Ala sehr schnell (vgl. Sopata 2010, 2011 zum schnellen Erwerb von anderen Bereichen des Deutschen als Zweitsprache durch Kinder in dem Alter). In KM8 und KM9 liegt der Gebrauch der Nullobjekte in der Topik-Position bei Ala bei über 20\%. Der Wert ist gleich der Häufigkeit des Gebrauchs der Nullobjekte in der Topik-Position in der letzten Sprachentwicklungsstufe bei Elisa, die Deutsch als Erstsprache erwirbt und die zu diesem Zeitpunkt 3;4 Jahre alt ist. Ala macht also schon nach 8 Kontaktmonaten zu Deutsch Gebrauch von Strukturen, die in der Erwachsenensprache und in der fortgeschrittenen Phase des Erstspracherwerbs vorkommen.

In Bezug auf die dritte Forschungsfrage zeigen die Ergebnisse der Untersuchung auf, dass der Erwerb der syntaktischen Einschränkungen für den Gebrauch der Nullobjekte im Erwerb der Zweitsprache dadurch erschwert ist, dass sie sich von denen in der Erstsprache des Kindes unterscheiden. Die Grundlage dieser Behauptung bilden die Daten zum Gebrauch der Nullobjekte in der NichtTopik-Position bei Ala. Im Polnischen sind Nullobjekte sowohl in der Topik-als auch in der Nicht-Topik-Position zulässig. Im Deutschen kommen sie nur in der Topik-Position vor. Die Nullobjekte in der Nicht-Topik-Position tauchen nicht in der deutschen Erwachsenensprache und auch sehr selten während der Sprachentwicklung des Deutschen als Erstsprache auf. Der hohe Anteil der Nullobjekte in der Nicht-Topik-Position in Alas Daten ist daher als Folge des Einflusses ihrer Erstsprache Polnisch auf die Entwicklung ihrer Zweitsprache Deutsch zu interpretieren.

Der Verlauf des Erwerbs der Fähigkeit, syntaktische Informationen in einen adäquaten Diskursrahmen zu setzen, ist also davon abhängig, ob er ein Teil des monolingualen oder des sukzessiven bilingualen Spracherwerbs ist. In beiden Situationen lernen aber die Kinder ziemlich schnell das Zusammenspiel von Diskurs und Syntax im Sprachgebrauch. Sie eignen sich die Fähigkeit an, den kognitiven Status des gegebenen referentiellen Arguments richtig festzulegen und sie treffen ziemlich schnell normgerechte Entscheidungen über die Form des Arguments, die im gegebenen Kontext zu verwenden ist. Da sie meistens in der fortgeschrittenen Stufe der Sprachentwicklung eine richtige Entscheidung treffen, heißt es, dass sie die Stelle des Arguments in der Gegebenheitshierarchie 
zu diesem Zeitpunkt wahrheitsgemäß bestimmt haben. Der richtige Gebrauch der Nullobjekte in der fortgeschrittenen Phase des Erst- und des frühen Zweitspracherwerbs bedeutet aber auch, dass die Kinder die syntaktischen Bedingungen für den Gebrauch der Nullargumente im Deutschen erkannt haben.

Die Daten der vorliegenden Untersuchung können schwer im Rahmen der Ansätze erklärt werden, die die Nullargumente auf die Komplexität der Verarbeitung der klitischen Elemente zurückführen (Hamann et al. 1996; Gruter 2006; Gavarró et al. 2010), weil die ausgelassenen Objekte in vielen Fällen gar nicht Klitika sein können. Das Kind scheint eher bei der Interpretation der ausgelassenen Kategorien vor allem Diskursstrategien zu berücksichtigen. Die ausgelassenen Elemente werden möglicherweise durch Diskurs und nicht durch entsprechende syntaktische Operationen interpretiert, die in der Erwachsenensprache für die Interpretation der Nullelemente zuständig sind.

\section{LITERATURVERZEICHNIS}

Castilla, A. / Pérez-Leroux, A. (2010). Omissions and substitutions in Spanish object clitics: Developmental optionality as a property of the representational system. Language Acquisition, 17, 2-25.

Clark, E. (1985). The acquisition of Romance, with special reference to French. In: D. Slobin (Hrsg.), The crosslinguistic study of language acquisition: Vol. 1. The data (S. 687-782). Hillsdale NJ: Erlbaum.

Costa, J. / Lobo, M. / Silva, C. (2012). Which category replaces an omitted clitic? The case of European Portuguese. In: P. Larranaga / P. Guijarro-Fuentes (Hrsg.), Pronouns and clitics in early language (S. 105-130). Berlin: Mouton de Gruyter.

Fukumura, K. / van Gompel, R. (2011). The effects of animacy in the choice of referring expressions. Language and Cognitive Processes, 26 (10), 1472-1504.

Gavarró, A. / Torrens, V. / Wexler, K. (2010). Object clitic omission: two language types. Language Acquisition, 17, 192-219.

Grüter, T. (2006). Object clitics and null objects in the acquisition of French. Montréal: McGill University dissertation.

Gundel, J. / Hedberg, N. / Zacharski, N. (1993). Cognitive status and the form of referring expressions in discourse. Language, 69 (2), 274-307.

Hamann, C. (1996). Null arguments in German child language. Language Acquisition, 5 (3), 155-208.

Hamann, C. / Rizzi, L. / Frauenfelder, U. (1996). On the acquisition of subject and object clitics in French. In: H. Clahsen (Hrsg.), Generative approaches to language acquisition (S. 309-334). Amsterdam: Benjamins.

Holmberg, A. (2005). Is there a little pro? Evidence from Finnish. Linguistic Inquiry, 36, 533-564.

Huang, C.-T.J. (1984). On the distribution and reference of empty pronouns. Linguistic Inquiry, 15, 53-574.

Hughes, M. / Allen, S. (2013). The effect of individual discourse-pragmatic features on referential choice in child English. Journal of Pragmatics, 56, 15-30.

Jakubowicz, C. / Müller, N. / Kang, O. / Riemer, B. / Rigaut, C. (1996). On the acquisition of the pronominal system in French and German. In: A. Springfellow / D. Cahana-Amitay / E. Hughes / A. Zukowski (Hrsg.), Proceedings of the 20th BUCLD (S. 374-385). Somerville, MA: Cascadilla Press. 
Jakubowicz, C. / Müller, N. / Riemer, B. / Rigaut, C. (1997). The case of subject and object omissions in French and German. In: E. Hughes / M. Hughes / A. Greenhill (Hrsg.), Proceedings of the 21 Annual Boston University Conference on language development (S. 331-342). Somerville MA: Cascadilla Press.

Kotin, M.L. (2003). Die werden-Perspektive und die werden-Periphrase im Deutschen. Frankfurt a.M.: Peter Lang.

Matthews, D. / Lieven, E. / Theakston, A. / Tomasello, M. (2006). The effect of perceptual availability and prior discourse on young children's use of referring expressions. Applied Psycholinguistics, 27, 403-422.

Müller, N. / Hulk, A. (2001). Crosslinguistic influence in bilingual language acquisition: Italian and French as recipient languages. Bilingualism: Language and Cognition, 4, 1-21.

Mykhaylyk, R. / Sopata, A. (2016). Object pronouns, clitics and omissions in child Polish and Ukrainian. Applied Psycholinguistics, 37 (5), 1051-1082.

Pérez-Leroux, A. / Pirvulescu, M. / Roberge, Y. (2008). Null objects in child language: Syntax and the lexicon. Lingua, 118, 370-398.

Sigurðsson, H. (2011). Conditions on argument drop. Linguistic Inquiry, 42, 267-304.

Sopata, A. (2009). Erwerbstheoretische und glottodidaktische Aspekte des frühen Zweitspracherwerbs. Sprachentwicklung der Kinder im natürlichen und schulischen Kontext. Poznań: Wydawnictwo Naukowe UAM.

Sopata, A. (2010). Inflectional morphology in child second language acquisition. In: J. Costa / A. Castro / M. Lobo / F. Pratas (Hrsg.), Language acquisition and development. Prodeedings of GALA 2009 (S. 419-429). Cambridge: Cambridge Scholars Publishing.

Sopata, A. (2011). Placement of infinitives in successive child language acquisition. In: E. Rinke / T. Kupisch (Hrsg.), The development of grammar: Language acquisition and diachronic change Volume in honor of Jürgen M. Meisel (S. 105-121). Amsterdam: John Benjamins.

Sopata, A. (2016). Null objects in adult and child Polish: Syntax, discourse and pragmatics. Lingua 183, 186-106. DOI: http://dx.doi.org/10.1016/j.lingua.2016.05.007.

Sopata, A. (2017). Referenzielle Nullargumente im Erstspracherwerb. In: E. Żebrowska / M. Olpińska-Szkiełko / M. Latkowska (Hrsg.), Germanistische Forschung in Polen. Gegenstände und Methoden, Formen und Wirkungen (S. 69-80). Warszawa: Stowarzyszenie Germanistów Polskich.

Wexler, K. / Gavarro, A. / Torrens, V. (2004). Feature checking and clitic omission in child Catalan and Spanish. In: R. Bok-Bennema / B. Hollebrandse / B. Kampers-Mahne / P. Sleeman (Hrsg.), Romance language and linguistic theories: Selected papers from Going Romance 2002 (S. 253-269). Amsterdam: Benjamins.

Received: 31.08.2021; revised: 23.10.2021

\section{ALDONA SOPATA}

Uniwersytet im. Adama Mickiewicza w Poznaniu

sopata@amu.edu.pl

ORCID: 0000-0002-0733-4455 